\title{
Quantifying and Reducing the Effect of Calibration Error on Variability of PET/CT Standardized Uptake Value Measurements
}

\author{
Catherine M. Lockhart, Lawrence R. MacDonald, Adam M. Alessio, Wendy A. McDougald, Robert K. Doot, \\ and Paul E. Kinahan \\ Department of Radiology, University of Washington, Seattle, Washington
}

\begin{abstract}
The purpose of this study was to measure the errors introduced by regular calibration of PET/CT scanners and to minimize the effect of calibration error on standardized uptake value measurements. Methods: Global calibration factors from 2 PET/CT scanners were recorded for 3.5 and $1.8 \mathrm{y}$, comparing manufacturer-recommended protocols with modified protocols to evaluate error contributions due to operator-influenced procedures. Dose calibrator measurements were evaluated using National Institute of Standards and Technology-traceable sources. Results: Dose calibrator variability was less than $1 \%$, although there was a consistent bias. Global scaling variability was reduced from $6 \%$ to $4 \%$ for scanner 1 and from $11 \%$ to $4 \%$ for scanner 2 when quality assurance and quality control procedures were applied to the calibration protocol. When calibrations were done using a ${ }^{68} \mathrm{Ge} /{ }^{68} \mathrm{Ga}$ phantom, the variability for both scanners was reduced to approximately $3 \%$. Conclusion: Applying quality assurance and quality control procedures to scanner calibration reduces variability, but there is a still a residual longitudinal scanner variability of $3 \%-4 \%$. The procedures proposed here reduce the impact of operator error on scanner calibration and thereby minimize longitudinal variability in standarized uptake value measurements.
\end{abstract}

Key Words: PET quantitation; PET scanner calibration; SUV accuracy

J Nucl Med 2011; 52:218-224

DOI: 10.2967/jnumed.110.083865

I maging of cancer with combined PET and x-ray CT scanners has become a standard component of oncology diagnosis and staging $(1,2)$. With lung cancer, for example, PET/CT of ${ }^{18} \mathrm{~F}$-FDG uptake allows a more accurate detection of both nodal and distant forms of metastatic disease (3), and tumor stage is still the most important prognostic factor for predicting the survival of patients with non-small cell lung cancer (4). In addition, metabolic activity of cancerous cells may prove a more reliable indicator of therapeu-

Received Oct. 3, 2010; revision accepted Oct. 26, 2010.

For correspondence or reprints contact: Paul Kinahan, University of Washington, Rm. 222 FIS, Box 357987, Seattle, WA 98195-7987.

E-mail: kinahan@uw.edu

COPYRIGHT @ 2011 by the Society of Nuclear Medicine, Inc. tic response than changes in tumor size (5). In light of an increasing need for effective diagnostic tools, and more accurate assessment of treatment response, PET/CT is becoming more important as a quantitative monitor of individual response and an evaluation tool for new drug therapies (6-8).

Quantitatively accurate images of tracer uptake are possible with PET, but there is often an unknown global bias and variance (9). In the primary application of PET for clinical diagnosis and staging of cancer, qualitative image fidelity is of paramount importance and accurate global quantification is not necessary (10). However, inaccuracies in image quantification measures can significantly affect successful evaluation of therapeutic response (11).

The goal of PET scanners is to measure radioactive concentration - for example, units of $\mathrm{kBq} / \mathrm{mL}$ in a volumetric PET image. The measured concentration depends on the amount of activity injected and distribution inside the patient. The standardized uptake value (SUV) has become the commonly reported unit of uptake in clinical PET (12). The SUV is defined as

$$
\mathrm{SUV}=\frac{R}{d^{\prime} / \tilde{V}}
$$

where $R(\mathrm{kBq} / \mathrm{mL})$ is the radioactivity per volume measured by the PET scanner. The denominator normalizes for variations in the injected activity $d(\mathrm{MBq})$ and patient size, where $d^{\prime}$ is decay-corrected to the scan time measurement of $R$, and $\tilde{V}$ is a surrogate for the body volume into which the tracer is distributed. Patient weight $(\mathrm{kg})$ is often used as a surrogate for the volume of distribution, in which case the SUV units are $\mathrm{g} / \mathrm{mL}$. Because adipose tissue, with the exception of brown fat, does not normally take up significant amounts of ${ }^{18} \mathrm{~F}-\mathrm{FDG}$, the estimated lean body mass or body surface area are sometimes used instead of weight $(13,14)$.

The measured radioactivity concentration, $R$, is calculated as

$$
R=g \times r / t, \quad \text { Eq. } 2
$$

where $r$ is the average voxel value in scanner arbitrary units, $t$ is the scan acquisition time, and $g$ is a global scaling factor with units of $(\mathrm{kBq} / \mathrm{mL}) /($ scanner arbitrary units/s). The average voxel value is in scanner-specific arbitrary 
units because of the large number of scale factors arising from quantitative corrections, raw data compression (if used), and reconstruction algorithms. There are several sources of error (e.g., partial-volume errors) that affect Equation 1; these sources are beyond the scope of this study but are described in the recent review by Boellaard (9). In this study, we specifically evaluate the variability introduced by the global scale factor $g$ - that is, the scanner calibration process. Each manufacturer has a slightly different procedure and recommended frequency of calibration for estimating $g$. In general, the calibration process involves scanning a waterfilled uniform cylinder containing a known amount of ${ }^{18} \mathrm{~F}$. The calibration operation can be expressed as

$$
g=c \times \frac{a^{\prime} \times t_{C}}{r_{C} \times V},
$$

where $r_{c}$ is the average voxel value (in scanner arbitrary units) for a large region of the water-filled uniform cylinder, $t_{c}$ is the acquisition time for the calibration scan, and $a^{\prime}$ is the decay-corrected activity (kBq) injected into the uniform cylinder, which in turn has volume $V(\mathrm{~mL})$. The factor $c$ here includes all other global multiplicative effects, such as isotope branching ratios, voxel volumes, and reconstruction algorithm-dependent scale factors.

In principle, measuring $g$ corrects for global sensitivity variations. However, scanner calibrations typically occur quarterly or semiannually and so cannot compensate for variations in $g$ that occur on a time scale shorter than months. There is little information available on the timevarying behavior of $g$. Studies by Doot et al. show that over a period of minutes to a few hours, the global efficiency variation is less than $0.5 \%$ (15). We report on scanner variability over longer periods (e.g., quarterly), which, to our knowledge, has not been reported previously.

Figure 1 illustrates the effect of incorrect global calibration on SUVs estimated from a patient scan. In this case, the calibration factor of Equation 3 deviated by 19.8\% above the subsequently determined true value. The scan was reconstructed with the erroneous scale factor and was also processed again after the calibration procedure was repeated to correct the known error. The images show that the SUV in the scan reconstructed with the erroneous scale factor deviates from the SUV of the corrected scan by $19.8 \%$.

The dose calibrator is another potential source of error in global scaling of PET image values. A National Institute of Standards and Technology (NIST)-traceable standard for ${ }^{18} \mathrm{~F}$ was recently developed as a means of monitoring accuracy and precision in dose calibrator measurements of ${ }^{18} \mathrm{~F}$-labeled PET tracers (16). As much as a $10 \%$ shift in absolute calibration has been observed when comparing dose calibrator models from the 2 predominant manufacturers of dose calibrators (17). This intercalibrator variability is important for multisite studies and comparisons. To understand the significance of intracalibrator variability, we note that the dose calibrator is used to obtain 2 of the values in Equation 1: the scale factor $g$ and the patient dose $\left(d^{\prime}\right)$; therefore, any constant bias in the dose calibrator measurements will cancel out. The 2 dose calibrator readings, however, are performed on different days. Thus, it is important to understand the longitudinal variability of an individual dose calibrator used for both scanner calibration and patient SUV measurements and any potential differences in longitudinal drift of measurement biases between the dose calibrator and PET scanner.

As a third source of error, inspection of Equations 1 and 3 indicates many radioactivity, time, and weight values that need to be measured and recorded properly for proper SUV quantification of PET images. In other words, there is a substantial opportunity for operator errors. A survey of procedures finds 9-15 operator-dependent steps for both Equation 1 and Equation 3. Errors made in the estimation of the global scale factor $g$ (Eq. 3) will affect all subsequent patient images-for example, Figure 1.

In this paper, we investigate the sources of error in instrument calibration and explore a new procedure to minimize the effect that human procedural inconsistencies have on scanner stability and calculated activity-correction factors. We evaluated the precision of activity-correction factors over a multiyear period on 2 PET/CT scanners and implemented a new procedure to detect and correct errors in

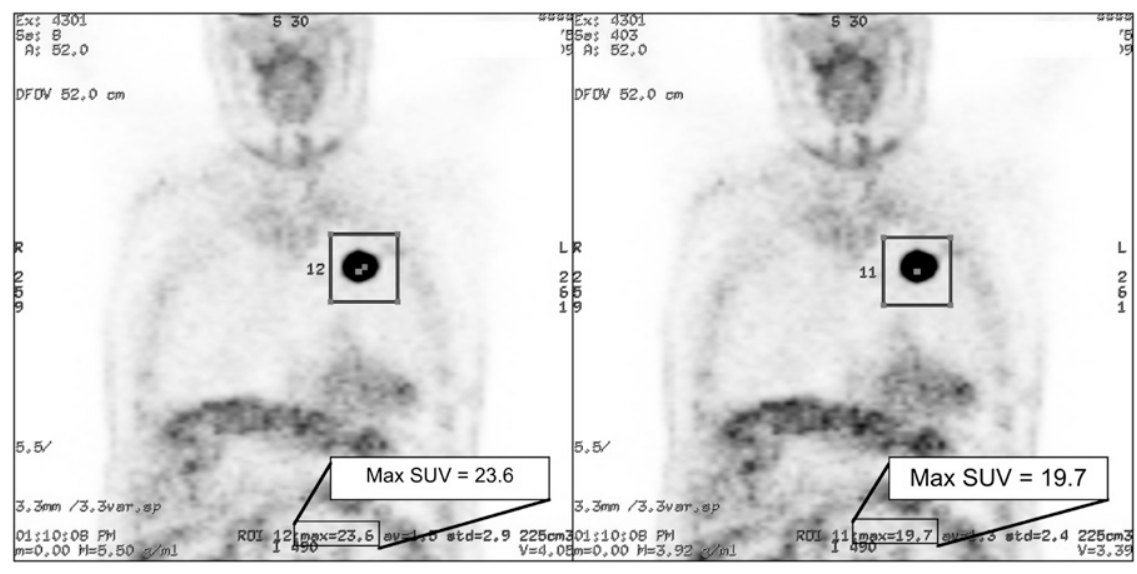

FIGURE 1. Patient scan reconstructed with erroneous scanner calibration scale factor (left) and again with corrected calibration (right). There is no visual difference between images, but erroneous SUV is $19.8 \%$ higher than corrected SUVs for highlighted region of interest (box). Max = maximum; $\mathrm{ROI}=$ region of interest. 
the calibration process. The goal was to preclude erroneous patient SUV measurements such as the ones shown in Figure 1.

\section{MATERIALS AND METHODS}

We evaluated the sources of calibration error on 2 of the same model of PET/CT scanner (Discovery STE; GE Healthcare) located in 2 different medical imaging centers. On these scanners, the global calibration factor, $g$ in Equations 2 and 3, is called the activity-calibration factor. The calibration factor variability for these scanners was evaluated using uniform cylinders filled with either ${ }^{18} \mathrm{~F}$ in water or ${ }^{68} \mathrm{Ge} /{ }^{68} \mathrm{Ga}$ in epoxy matrix.

\section{Scanner Calibration Measurements}

The manufacturer-recommended protocol comprises quarterly estimation of the calibration factor using a water-filled phantom (diameter, $20 \times 20 \mathrm{~cm}$ ) containing ${ }^{18} \mathrm{~F}(18)$ (half-life, $110 \mathrm{~min}$ (19)). In addition, we added a modified procedure using a phantom of the same size, with the longer-lived cylindric source (diameter, $20 \mathrm{~cm}$ ) containing ${ }^{68} \mathrm{Ge} /{ }^{68} \mathrm{Ga}$ in epoxy. In all cases with scanner measurements, the actual isotope used (either ${ }^{18} \mathrm{~F}$ or ${ }^{68} \mathrm{Ge} /{ }^{68} \mathrm{Ga}$ ) was entered into the examination acquisition parameters for appropriate compensation of the branching ratio (20). The halflife of ${ }^{68} \mathrm{Ge}$ is $270.8 \mathrm{~d}$, which decays to ${ }^{68} \mathrm{Ga}$-a positron emitter (half-life, $68.3 \mathrm{~min}$ ). The manufacturer of the ${ }^{68} \mathrm{Ge} /{ }^{68} \mathrm{Ga}$ phantom (Sanders Medical) reports an activity level accuracy of $\pm 10 \%$. The use of a ${ }^{68} \mathrm{Ge} /{ }^{68} \mathrm{Ga}$ phantom allowed us to calibrate the scanner with exactly the same source over the course of many months and thus eliminate variability of calibration source activity. Although a bias in the activity level exists, that bias remains the same while using the same phantom. We adhered to the manufacturer-recommended protocol, except that we used an increased frequency of measurement and added the ${ }^{68} \mathrm{Ge} /{ }^{68} \mathrm{Ga}$ phantom measurements, which were not used to calculate the calibration factors used for patient scans. Activity-correction factors were calculated and recorded for both 2-dimensional (2D) and 3-dimensional (3D) acquisition modes on the same day using the same calibration phantom.

An activity check was performed using the same ${ }^{68} \mathrm{Ge} /{ }^{68} \mathrm{Ga}$ calibration phantom. In this case, a slightly modified patient protocol, with a shorter scan time than prescribed clinically, was used to scan and reconstruct the phantom images. A cubic region of interest (ROI) was placed in the center of the image, sized as large as reasonably possible while remaining away from the edges of the active source volume to avoid resolution loss. From these ROI values, the activity concentration as measured by the scanner was recorded and compared with the reported value. If the reported activity was more that $5 \%$ different from the expected value, the calibration factors were recorded but not used for patient scans, and the calibration procedure was repeated.

The calibration factors were recorded over a 3.5-y period for scanner 1 and at 21 mo for scanner 2. Several calibration factors were identified as incorrect because of operator error and were rejected before any patient scans were obtained. The data were analyzed both with and without the known erroneous calibration factors.

\section{Dose Calibrator Measurements}

Serial dose calibrator measurements were taken using a NISTtraceable dose calibrator standard ${ }^{68} \mathrm{Ge} /{ }^{68} \mathrm{Ga}$ solid-matrix aliquot configured for measurements using the ${ }^{18} \mathrm{~F}$ setting with a typical dose calibrator syringe holder (as specified by the manufacturer, RadQual, Inc.). The same dose calibrator (Capintec-127R) was used for all ${ }^{18} \mathrm{~F}$ dose assays injected into the calibration phantom. Measurements were recorded with dose calibrator settings for both ${ }^{68} \mathrm{Ge} /{ }^{68} \mathrm{Ga}$ and ${ }^{18} \mathrm{~F}$ (using the ${ }^{68} \mathrm{Ge} /{ }^{68} \mathrm{Ga}$ source) and compared with the expected values calculated from standard exponential decay (half-life, 270.8 d) from a known initial value of 19.5 $\mathrm{MBq} \pm 1.31 \%$. In addition to the serial measurements using the NIST-calibrated ${ }^{68} \mathrm{Ge} /{ }^{68} \mathrm{Ga}$ aliquot, daily constancy measurements performed by nuclear medicine technologists were extracted for 3 $y$ as another measure of dose calibrator variation over time. The daily measurements were done using a NIST-traceable ${ }^{137} \mathrm{Cs}$ standard source placed in the syringe holder of the dose calibrator. We evaluated the percentage deviation from the expected value using the decay rate of the radioactive source from the nominal initial value provided by the manufacturer.

\section{RESULTS}

Figure 2 shows variations in the scanner calibration values generated using both ${ }^{18} \mathrm{~F}$ and ${ }^{68} \mathrm{Ge} /{ }^{68} \mathrm{Ga}$ source cylinders collected from 2 scanners in 2D and 3D modes. Outlier values due to identified operator error are highlighted. The operator errors were distributed throughout all steps in the process of measurement, data recording, and data entry that are involved in scanner calibration. Histograms of all scanner calibration values are shown in Figure 3 for scanner 1.

Figure 3 shows the distribution of measured calibration factors from scanner 1 . The mean calibration factors differ between ${ }^{18} \mathrm{~F}$ and ${ }^{68} \mathrm{Ge} /{ }^{68} \mathrm{Ga}$ by approximately $8.5 \%$ for both 2D and 3D calibration scans. This is most likely due to a bias in the manufacturer-reported activity value of the ${ }^{68} \mathrm{Ge} /{ }^{68} \mathrm{Ga}$ source. Means, SD, and coefficients of variation were calculated with and without known outlier values for both scanners (Table 1). The 95\% confidence interval for the coefficient of variation for each system was calculated assuming a normal distribution. In addition, the maximum observed change in the scanner calibration value between 2 consecutive calibrations was reported to illustrate the maximum potential change before and after calibration.

Activity-correction factors for manufacturer-recommended ${ }^{18} \mathrm{~F}$-based calibration scans vary by approximately $6 \%$ for scanner 1 and approximately $11 \%$ for scanner 2 , when outlying points due to known operator error are included in the data. By removing the known erroneous points, shown in Figure 2, the variability is reduced to approximately $4 \%$ for both scanners. When the protocol is performed with a ${ }^{68} \mathrm{Ge} /{ }^{68} \mathrm{Ga}$ phantom, eliminating the phantom loading steps required with the ${ }^{18} \mathrm{~F}$ water phantom, the variability is reduced to approximately $2 \%-3 \%$ in all cases.

Activity concentration measured using a ${ }^{68} \mathrm{Ge} /{ }^{68} \mathrm{Ga}$ phantom with a protocol similar to a standard patient scan were compared with the expected activity concentration based on calculated exponential decay. In all cases, activity concentrations for both 2D and 3D scans were consistently greater than the expected value based on the manufacturerquoted nominal value of the ${ }^{68} \mathrm{Ge} /{ }^{68} \mathrm{Ga}$ phantom. Visually, the scanner measurements appear to trend generally with the expected decay of ${ }^{68} \mathrm{Ge}$ as shown in Figure 4. 

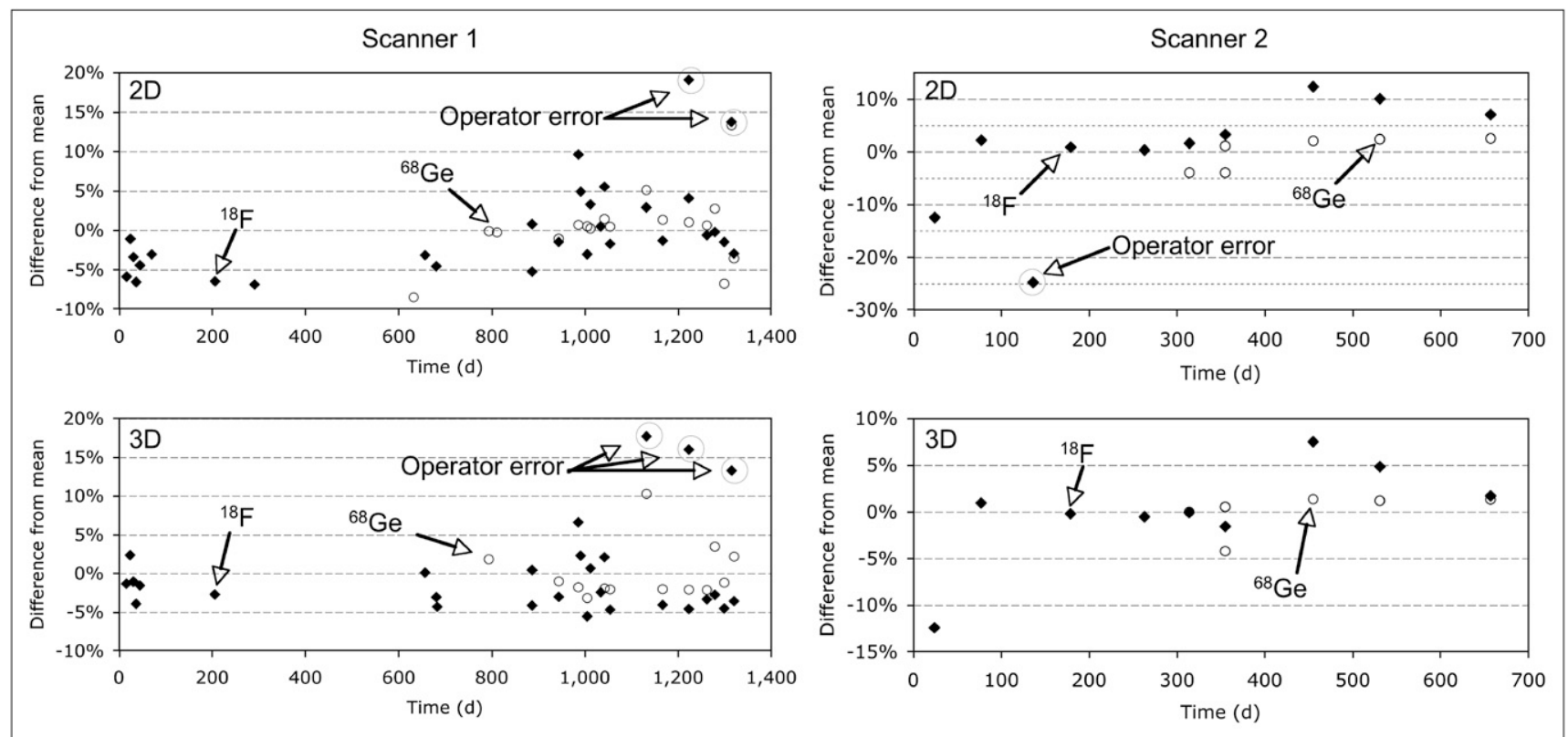

FIGURE 2. Percentage deviation of scanner calibration values for $2 \mathrm{D}$ and $3 \mathrm{D}$ activity-correction factors for 2 scanners with both ${ }^{18} \mathrm{~F}$ and ${ }^{68} \mathrm{Ge} /{ }^{68} \mathrm{Ga}$ sources.

Another potential source of calibration error is from variability in the dose calibrator. Figure 5 shows serial measurements of the NIST-traceable ${ }^{68} \mathrm{Ge} /{ }^{68} \mathrm{Ga}$ aliquot in the syringe holder of the dose calibrator.

The expected value was calculated using the standard exponential decay of ${ }^{68} \mathrm{Ge}$ from a known nominal value provided by the manufacturer. Activity present in the ${ }^{68} \mathrm{Ge} /{ }^{68} \mathrm{Ga}$ aliquot was measured in the dose calibrator and compared with calculated values on the same date. The measured values have a positive bias but appear to follow a consistent trend over time and do not change significantly over the life of the aliquot source, consistent with a fixed bias.

Dose calibrator variability was evaluated using daily constancy measurements with a NIST-traceable ${ }^{137} \mathrm{Cs}$ calibration source (Fig. 6). The measurements recorded over the past 3 y have an SD of $0.90 \%$.

\section{DISCUSSION}

The SUV is a measure of relative radiotracer uptake in tissue. In PET, SUV is increasingly used to monitor the effects of treatment or to evaluate serial patient studies in single-center and multicenter trials. Several studies have shown that the best-case short-term test-retest SUV variability was approximately $10 \%$ in patients (21-26). Geworski et al. evaluated the accuracy of a group of PET scanners at a single time point (27). To our knowledge, however, there have been no studies evaluating long-term variability of the PET SUVs due to instrumentation effects and operator error.

Our results show that there is an approximately $3 \%$ additional long-term variability that is intrinsic to the PET scanners (based on the ${ }^{68} \mathrm{Ge} /{ }^{68} \mathrm{Ga}$ phantom data). The longterm variability increases to approximately $4 \%$ when ${ }^{18} \mathrm{~F}$ filled phantoms are used. Calibration factors were calculated
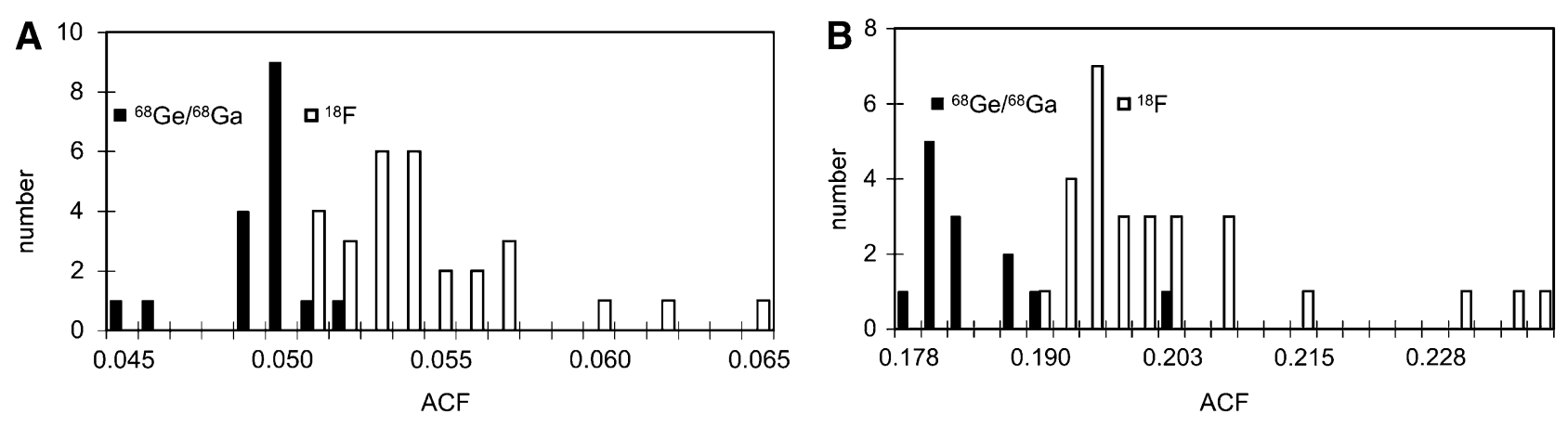

FIGURE 3. Histograms of scanner calibration values for scanner 1 generated using ${ }^{18} \mathrm{~F}$ and ${ }^{68} \mathrm{Ge} /{ }^{68} \mathrm{Ga}$ for $2 \mathrm{D}(\mathrm{A})$ and $3 \mathrm{D}(\mathrm{B})$ acquisition modes. ACF = activity-correction factor. 
TABLE 1

Statistical Analysis of Calibration Values for 2 Scanners, With and Without Outliers Due to Known Operator Error

\begin{tabular}{|c|c|c|c|c|c|c|}
\hline Scanner & Data set & $n$ & Mean & $\begin{array}{c}\text { Coefficient } \\
\text { of variation (\%) }\end{array}$ & $\begin{array}{l}95 \% \text { confidence interval range } \\
\text { for coefficient of variation }\end{array}$ & $\begin{array}{l}\text { Maximum } \\
\text { change (\%) }\end{array}$ \\
\hline \multirow[t]{6}{*}{1} & ${ }^{18} \mathrm{~F} 2 \mathrm{D}$-all data & 29 & 0.054 & 6.1 & $4.8-8.2$ & 20.3 \\
\hline & ${ }^{18} \mathrm{~F} 2 \mathrm{D}$-outliers removed & 27 & 0.053 & 4.2 & $3.3-5.7$ & 11.2 \\
\hline & ${ }^{68} \mathrm{Ge} /{ }^{68} \mathrm{Ga} 2 \mathrm{D}$ —all data & 17 & 0.049 & 3.2 & $2.4-4.9$ & 9.5 \\
\hline & ${ }^{18} \mathrm{~F} 3 \mathrm{D}$-all data & 28 & 0.201 & 6.2 & $4.9-8.4$ & 22.4 \\
\hline & ${ }^{18} \mathrm{~F} 3 \mathrm{D}$-outliers removed & 25 & 0.197 & 3.0 & $2.3-4.1$ & 9.8 \\
\hline & ${ }^{68} \mathrm{Ge} /{ }^{68} \mathrm{Ga} 3 \mathrm{D}$-all data & 13 & 0.183 & 3.7 & $2.6-6.1$ & 12.3 \\
\hline \multirow[t]{6}{*}{2} & ${ }^{18} \mathrm{~F} 2 \mathrm{D}$-all data & 10 & 0.050 & 11.0 & $7.6-20.1$ & 25.7 \\
\hline & ${ }^{18} \mathrm{~F} 2 \mathrm{D}$-outliers removed & 8 & 0.053 & 4.3 & $2.9-8.8$ & 8.7 \\
\hline & ${ }^{68} \mathrm{Ge} /{ }^{68} \mathrm{Ga} 2 \mathrm{D}$-all data & 6 & 0.051 & 3.1 & $1.9-7.6$ & 5.0 \\
\hline & ${ }^{18} \mathrm{~F}$ 3D—all data & 9 & 0.194 & 5.5 & $2.0-6.2$ & 13.3 \\
\hline & ${ }^{18} \mathrm{~F} 3 \mathrm{D}$-outliers removed & 8 & 0.197 & 3.0 & $2.0-6.2$ & 9.1 \\
\hline & ${ }^{68} \mathrm{Ge} /{ }^{68} \mathrm{Ga} 3 \mathrm{D}$-all data & 4 & 0.187 & 2.1 & $1.3-5.3$ & 4.7 \\
\hline
\end{tabular}

Units are ([scanner arbitrary units] $\times \mathrm{s} / \mathrm{kBq} \times \mathrm{mL})$.

for both $2 \mathrm{D}$ and $3 \mathrm{D}$ acquisition modes on the same day, using the same calibration phantom, and the associated variability in these factors was consistent (within $95 \%$ confidence intervals).

A first-order evaluation of error propagation in Equation 1 yields the uncertainties, adding in quadrature (28)

$$
\frac{\delta \mathrm{SUV}}{\mathrm{SUV}}=\sqrt{\left(\frac{\delta g}{g}\right)^{2}+\left(\frac{\delta r}{r}\right)^{2}+\left(\frac{\delta t}{t}\right)^{2}+\left(\frac{\delta \tilde{V}}{\tilde{V}}\right)^{2}+\left(\frac{\delta d^{\prime}}{d^{\prime}}\right)^{2}} \cdot \text { Eq. } 4
$$

Thus, a $4 \%$ variability $(\delta g / g)$ in the scanner calibration factor is negligible when compared with the $10 \%$ test-retest variability in patients $(\delta r / r)$. This low level of variability, however, assumes that quality assurance and quality control (QA/QC) procedures are applied to the calibration process to remove errors. If there are errors in the calibration process, then the long-term variability can easily exceed $10 \%$,

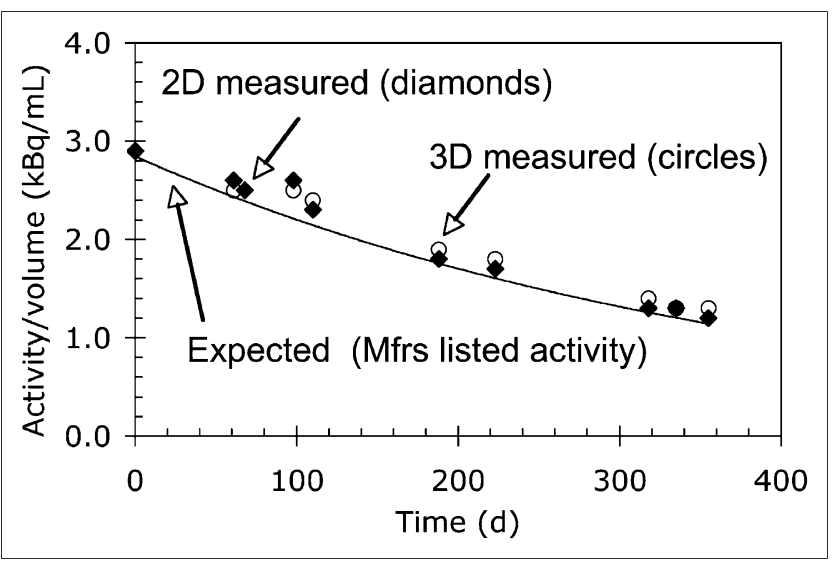

FIGURE 4. Results of activity check for scanner 1 compared with expected decay based on nominal value quoted by manufacturer. Mfrs = manufacturers. substantially increasing the overall variability of patient SUV measurements, because there is no upper limit on the error that can be introduced by the calibration process. In some cases, the change in SUVs due to the calibration process exceeded 20\%. Errors in PET activity concentration calibration procedures have a proportional effect on SUV calculation.

At present, the manufacturer's recommended calibration procedures typically require periodic calibration. In our study, the detected errors were distributed over the steps of the scanner calibration process with no consistent pattern. These steps include phantom preparation, data measurement, data recording, and data entry. There are no data we are aware of on the frequency and magnitude of these types of errors in general practice, but anecdotal evidence indicates that such errors do occur at many PET centers.

The calibration procedure to generate the activitycorrection factors involves human interaction, suggesting

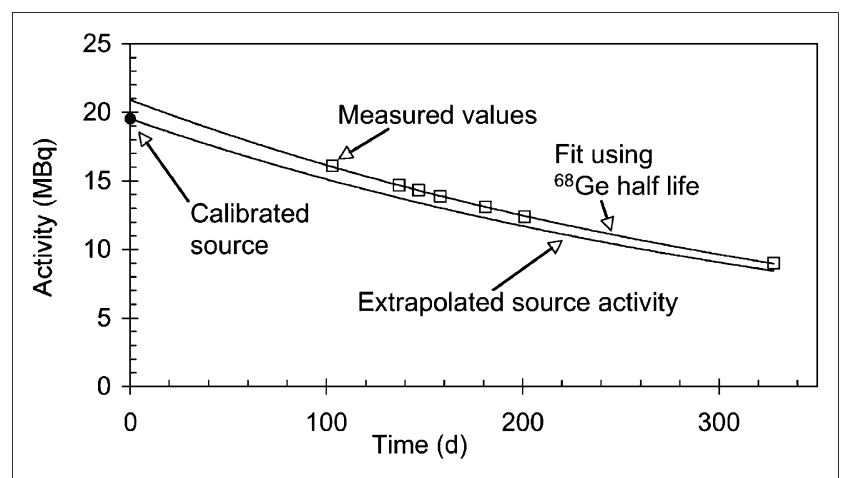

FIGURE 5. Dose calibrator measurements of NIST-traceable ${ }^{68} \mathrm{Ge} /{ }^{68} \mathrm{Ga}$ aliquot used for ${ }^{18} \mathrm{~F}$ dose calibration. Curve through measured data points is exponential fit. Expected curve is based on manufacturer's recommended settings for ${ }^{18} \mathrm{~F}$ sources for dose calibrator used. 


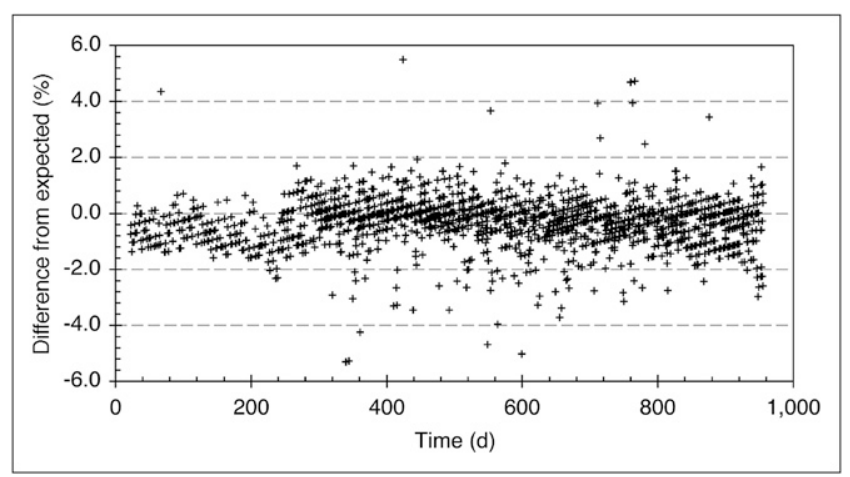

FIGURE 6. Daily constancy values measured using NIST-traceable ${ }^{137} \mathrm{Cs}$ calibration source with dose calibrator used for clinical PET dose assay. Sample SD is less than $0.90 \%$ over $3-y$ period.

a greater potential for error and leading to variability in scanner performance. The overall variability in activitycorrection factors for both scanners is reduced when the ${ }^{68} \mathrm{Ge} /{ }^{68} \mathrm{Ga}$ phantom is used instead of an operator-prepared ${ }^{18} \mathrm{~F}$ water phantom, suggesting that either operator error or error in dose calibration may account for $1 \%-3 \%$ of the overall variability, after known errors are removed. However, we do not recommend the use of a ${ }^{68} \mathrm{Ge} /{ }^{68} \mathrm{Ga}$ phantom instead of a standard ${ }^{18} \mathrm{~F}$ water phantom, because this would not compensate for changes in the dose calibrator measurements. A fixed ${ }^{68} \mathrm{Ge} /{ }^{68} \mathrm{Ga}$ phantom is useful, however, as a calibration source that is independent of the scanner-calibration procedure. In other words, a postcalibration activity check should not be performed with the same source used to generate the calibrations.

The SD of dose calibrator measurements in our study is less than $1 \%$. There is an overall bias in the measured activity, and a similar bias has been seen in all dose calibrators tested (17). As noted, however, a constant bias will not affect SUV measurements if the same dose calibrator is used for scanner calibration and patient scans. However, recent studies by Zimmerman et al. (17) and Doot et al. (29) have indicated that dose calibrator bias is not always constant. In addition, if different dose calibrators are used, or if a ${ }^{68} \mathrm{Ge} /{ }^{68} \mathrm{Ga}$ phantom is used for scanner calibration, then consistent reference sources should be used with the dose calibrators (17).

\section{Recommendations}

On the basis of this work, we recommend adding the following $2 \mathrm{QA} / \mathrm{QC}$ procedures to routine clinical calibration:

1. Verifying that the scanner's global calibration factor does not change by more than a small amount (e.g., 5\%).

2. Testing the scanner performance by repeating a scan of a known source (e.g., test phantom) after the scanner calibration process, and verifying that the measured value also does not change by more than a small amount (e.g., 5\%).

However, it is possible that the scanner's true efficiency might change by more than, for example, $5 \%$. In this case, the scanner calibration factor should shift by the same relative amount. This should be verified by repeated testing.

In principle, either procedure 1 or procedure 2 would be sufficient. Both are recommended, however, because procedure 1 determines the global calibration factor directly, whereas procedure 2 is a more direct measure of the impact on clinical images. A summary of the enhanced calibration process flow of measurements with QA/QC checks is listed in Table 2.

The results presented here are for 2 PET/CT scanners of the same type. Each manufacturer (or even different scanners from the same manufacturer) will have variations of the calibration procedures used in this study. However, the main results (long-term variability $>3 \%$ and the value of QA/QC procedures applied to calibration) apply equally to all PET scanners.

TABLE 2

Typical Calibration Procedure and Recommended Additions

\begin{tabular}{|c|c|}
\hline Step & Procedure \\
\hline 1 & Assay syringe with ${ }^{18} \mathrm{~F}$. Record activity and time. \\
\hline 2 & Inject and mix ${ }^{18} \mathrm{~F}$ in water-filled $20-\mathrm{cm}$-diameter cylinder. \\
\hline 3 & Reassay syringe for residual activity. Record activity and time. \\
\hline 4 & Scan phantom, reconstruct image, and measure average value per milliliter from large ROI. \\
\hline 5 & $\begin{array}{l}\text { Compute new scanner calibration factor ( } g \text { in Eq. 3) as ratio of ROI average to net concentration in phantom after decay } \\
\text { correction. }\end{array}$ \\
\hline $6^{*}$ & Compare new scanner calibration factor with average of prior scanner calibration factors. \\
\hline $7^{\star}$ & If there is more than a small difference (e.g., $5 \%)$, check all measurement and data entry steps, or repeat steps $1-6$. \\
\hline 8 & Install calculated scale factor as new scanner calibration for subsequent patient scans. \\
\hline $9^{*}$ & $\begin{array}{l}\text { Scan a known test object (e.g., }{ }^{68} \mathrm{Ge} /{ }^{68} \mathrm{Ga} \text { phantom) using standard clinical protocol and measure } \mathrm{kBq} / \mathrm{mL} \text { on reconstructed } \\
\text { image. }\end{array}$ \\
\hline $10^{*}$ & $\begin{array}{l}\text { If there is more than a small difference in measured activity from previous images of test object (e.g., } 5 \% \text { ), check all } \\
\text { measurement and data entry steps, or repeat steps } 1-8 \text {. }\end{array}$ \\
\hline
\end{tabular}

*Recommended additions to typical calibration procedures. 


\section{CONCLUSION}

SUV variability and bias may be introduced by global scale factors due to inherent system variability, human procedural error, and inconsistencies in the dose calibrator used to assay calibration radioactivity doses. We presented a procedure whereby the operator-filled ${ }^{18} \mathrm{~F}$ phantom is augmented by a similar cylinder containing a long-lived isotope of ${ }^{68} \mathrm{Ge} /{ }^{68} \mathrm{Ga}$. This addition checks for operator errors from the dose assay and phantom-filling steps and was shown to reduce variations in global scale factors. This process, however, does not include cross-calibration with a dose calibrator.

The protocol presented here is designed to identify errors before they can be perpetuated through patient scans. Our data suggest that even by following the protocol as consistently as possible, and correcting any errors before they are incorporated into clinical scans, there is a 3\%-4\% variability introduced by activity-correction factors over time, reflecting the typical long-term variability of the scanner if QA/QC procedures are applied to scanner calibration. If $\mathrm{QA} / \mathrm{QC}$ procedures are not applied, which is common practice, then SUV variability can be much larger.

\section{ACKNOWLEDGMENTS}

We thank Drs. Jay Williams and Steve Ross (GE Healthcare) for their help with data analysis, Dr. Osama Mawlawi (MD Anderson Cancer Center) for help with data extraction from the systems under study, and the nuclear medicine technologists from University of Washington Medical Center and Seattle Cancer Care Alliance for their patience and support. This study was supported by NCI (SAIC) contract 24XS036-004; NIH grants R01-CA74158, R01-CA115870, and U01-CA148131; and a research contract from GE Healthcare.

\section{REFERENCES}

1. Fletcher JW, Djulbegovic B, Soares HP, et al. Recommendations on the use of ${ }^{18}$ F-FDG PET in oncology. J Nucl Med. 2008;49:480-508.

2. Weber WA, Grosu AL, Czernin J. Technology insight: advances in molecular imaging and an appraisal of PET/CT scanning. Nat Clin Pract Oncol. 2008;5:160-170.

3. Hicks RJ, Lau E, Alam NZ, Chen RY. Imaging in the diagnosis and treatment of non-small cell lung cancer. Respirology. 2007;12:165-172.

4. Brundage MD, Davies D, Mackillop WJ. Prognostic factors in non-small cell lung cancer: a decade of progress. Chest. 2002;122:1037-1057.

5. Weber WA. Assessing tumor response to therapy. J Nucl Med. 2009;50(suppl 1):1S-10S.

6. Frank R, Hargreaves R. Clinical biomarkers in drug discovery and development. Nat Rev Drug Discov. 2003;2:566-580.
7. Kelloff GJ, Hoffman JM, Johnson B, et al. Progress and promise of FDG-PET imaging for cancer patient management and oncologic drug development. Clin Cancer Res. 2005;11:2785-2808.

8. Wahl RL, Jacene H, Kasamon Y, Lodge MA. From RECIST to PERCIST: evolving considerations for PET response criteria in solid tumors. J Nucl Med. 2009;50(suppl 1):122S-150S.

9. Boellaard R. Standards for PET image acquisition and quantitative data analysis. J Nucl Med. 2009;50(suppl 1):11S-20S.

10. Coleman RE. Is quantitation necessary for oncological PET studies? Eur J Nucl Med Mol Imaging. 2002;29:133-135.

11. Lammertsma AA, Hoekstra CJ, Giaccone G, Hoekstra OS. How should we analyse FDG PET studies for monitoring tumour response? Eur J Nucl Med Mol Imaging. 2006;33:16-21.

12. Thie JA. Understanding the standardized uptake value, its methods, and implications for usage. J Nucl Med. 2004;45:1431-1434.

13. Kim CK, Gupta N, Chandramouli B, Alavi A. Standardized uptake values of FDG: body surface area correction is preferable to body weight correction. J Nucl Med. 1994;35:164-167.

14. Zasadny KR, Wahl RL. Standardized uptake values of normal tissues at PET with 2-[fluorine-18]-fluoro-2-deoxy-D-glucose: variations with body weight and a method for correction. Radiology. 1993;189:847-850.

15. Doot RK, Scheuermann JS, Christian PE, Karp JS, Kinahan PE. Instrumentation factors affecting variance and bias of quantifying tracer uptake with PET/CT. Med Phys. 2010;37:6035.

16. Zimmerman BE, Cessna JT. Development of a traceable calibration methodology for solid ${ }^{68} \mathrm{Ge} /{ }^{68} \mathrm{Ga}$ sources used as a calibration surrogate for ${ }^{18} \mathrm{~F}$ in radionuclide activity calibrators. $J$ Nucl Med. 2010;51:448-453.

17. Zimmerman B, Kinahan P, Galbraith W, Allberg K, Mawlawi O. Multicenter comparison of dose calibrator accuracy for PET imaging using a standardized source [abstract]. J Nucl Med. 2009;50(suppl 2):472.

18. GE Healthcare. Well counter correction. Discovery ST, STE, \& RX HP60 Installation Manual. Direction 5141129-100. Piscataway, New Jersey: GE Healthcare; 2006.

19. Garcia-Torano E, Medina VP, Ibarra MR. The half-life of ${ }^{18} \mathrm{~F}$. Appl Radiat Isot. 2010;68:1561-1565, discussion 1565 .

20. Cherry SR, Sorenson JA, Phelps ME. Physics in Nuclear Medicine. Philadelphia, PA: Saunders; 2003.

21. Krak NC, Boellaard R, Hoekstra OS, Twisk JW, Hoekstra CJ, Lammertsma AA. Effects of ROI definition and reconstruction method on quantitative outcome and applicability in a response monitoring trial. Eur J Nucl Med Mol Imaging. 2005;32:294-301

22. Minn H, Zasadny KR, Quint LE, Wahl RL. Lung cancer: reproducibility of quantitative measurements for evaluating 2-[F-18]-fluoro-2-deoxy-D-glucose uptake at PET. Radiology. 1995;196:167-173.

23. Nahmias C, Wahl LM. Reproducibility of standardized uptake value measurements determined by ${ }^{18}$ F-FDG PET in malignant tumors. J Nucl Med. 2008; 49:1804-1808.

24. Nakamoto Y, Zasadny KR, Minn H, Wahl RL. Reproducibility of common semiquantitative parameters for evaluating lung cancer glucose metabolism with positron emission tomography using 2-deoxy-2-[ $\left.{ }^{18} \mathrm{~F}\right]$ fluoro-D-glucose. Mol Imaging Biol. 2002;4:171-178.

25. Velasquez LM, Boellaard R, Kollia G, et al. Repeatability of ${ }^{18}$ F-FDG PET in a multicenter phase I study of patients with advanced gastrointestinal malignancies. J Nucl Med. 2009;50:1646-1654.

26. Weber WA, Ziegler SI, Thodtmann R, Hanauske AR, Schwaiger M. Reproducibility of metabolic measurements in malignant tumors using FDG PET. $J$ Nucl Med. 1999;40:1771-1777.

27. Geworski L, Knoop BO, de Wit M, Ivancevic V, Bares R, Munz DL. Multicenter comparison of calibration and cross calibration of PET scanners. J Nucl Med. 2002;43:635-639.

28. Bevington P, Robinson DK. Data Reduction \& Error Analysis for the Physical Sciences. Dubuque, IA: McGraw Hill Publishing Company; 2003.

29. Doot R, Allberg K, Kinahan P. Errors in serial PET SUV measurements [abstract]. J Nucl Med. 2010;51(suppl 2):126. 